\title{
The Effect of Socioeconomic Class and Immigrant Status on Disease Activity in Rheumatoid Arthritis: Data from BARFOT, a Multi-Centre Study of Early RA
}

\author{
Maria L.E. Andersson ${ }^{1}$, Stefan Bergman ${ }^{3}$, Maria K. Söderlin ${ }^{*, 2,3}$ for the BARFOT Study Group ${ }^{\S}$ \\ ${ }^{1}$ Research and Development Centre, Spenshult Rheumatology Hospital, Oskarström, Sweden \\ ${ }^{2}$ Karlskrona Central Hospital, Karlskrona, Sweden \\ ${ }^{3}$ Department of Rheumatology, IKVL, Lund University, Lund, and Research and Development Centre, Spenshult \\ Rheumatology Hospital, Oskarström, Sweden
}

\begin{abstract}
Background: There have been no reports on the effect of immigrant status and socioeconomic status on outcome in rheumatoid arthritis (RA) in Sweden.

Methods: Between 1992 and 2006, 2,800 patients were included in the BARFOT study on early RA in Sweden. Disease Activity Score 28 joints (DAS28), Health Assessment Questionnaire (HAQ), treatment and European League Against Rheumatism (EULAR) response criteria were registered. In 2010, 1,430 patients completed a questionnaire enquiring about demographics and lifestyle factors.

Results: One hundred and thirty-nine of the 1,430 patients $(9.7 \%)$ were immigrants. At baseline immigrants had higher mean HAQ (1.2 vs 0.97 for non-immigrants, $\mathrm{p}=0.001)$, DAS28 (5.6 vs 5.2, $\mathrm{p}=0.000)$, visual analog scale (VAS) pain (56 $\mathrm{mm}$ vs $45 \mathrm{~mm}, \mathrm{p}=0.000)$, VAS global health $(53 \mathrm{~mm} v s 44 \mathrm{~mm}, \mathrm{p}=0.000)$ and tender joint count (TJC) $(10$ vs $8, \mathrm{p}=0.000)$. These differences persisted for up to 2 years of follow-up (for HAQ, for up to 8 years of follow-up). Immigrant status did not have any effect on swollen joint count (SJC), ESR, CRP or EULAR response. Socioeconomic class did not have any effect on treatment or outcome.

Conclusions: Immigrants scored worse in pain, function and TJC for up to 2 years of follow-up, but they did not differ from non-immigrants in objective measures of inflammation or EULAR outcome. This could be due to different perceptions of health and pain and/or the stress of immigration. Socioeconomic class had no effect on treatment or outcome, and this could be due to the relatively egalitarian society in Sweden.
\end{abstract}

Keywords: Rheumatoid arthritis, socioeconomic class, immigrants, epidemiology.

\section{INTRODUCTION}

Studies from Canada, the Netherlands and the UK have reported a negative effect of lower socioeconomic status on prognosis in rheumatoid arthritis (RA) patients [1-4]. An earlier study from Sweden reported that hospital care for RA patients was associated with certain occupations such as farmers, miners and quarry workers, electrical workers, other construction workers, and engine and motor operators for men, and for women assistant nurses, and religious, juridical and other social-science-related workers [5]. An educational

*Address correspondence to this author at the Department of Internal Medicine, Karlskrona Central Hospital 37185, Karlskrona, Sweden;

Tel: +46-455-734691; Fax: +46-455-736868;

E-mail: maria.soderlin@ltblekinge.se

${ }^{\S}$ Members of the BARFOT study group: Sofia Ajeganova, Maria Andersson, Valentina Bala, Stefan Bergman, Ann Bremander, Kristina Forslind, Ingiäld Hafström, Catharina Keller, Ido Leden, Bengt Lindell, Karina Malm, Ingemar Petersson, Björn Svensson, Maria Söderlin, Annika Teleman, Jan Theander, and Anneli Östenson. level of > 12 years was found to be associated with less hospitalisation in Sweden [5]. Studies from the US have shown minority health disparities [6] and that ethnicity, immigration and non-English language skills had a negative effect on outcome in RA in a university clinic setting [7].

In Sweden, medical care is universally available and easily accessible. Most rheumatologists in Sweden are employed in public hospitals, i.e. hospitals run by the county councils. Treatment of RA in Sweden does not depend on whether the patient seeks care in hospitals in the public sector or in the private sector. The cost of healthcare and treatment for patients is low in the public sector, and private rheumatologists' fees for the patients are comparable to these. Access to anti-rheumatic treatment and care in Sweden is not dependent on employment status or on whether or not the patient is privately insured. Prescription drugs are subsidised by the state and are free of charge above 2,200 SEK $(\approx € 220)$ annually (based on costs in 2013).

No formal level of disease activity in RA is required by the Swedish Society for Rheumatology guidelines for biological treatment. However, the guidelines state that the 
patients should have active disease and should have previously received methotrexate (MTX) alone or in combination with other disease-modifyíng anti-rheumatic drugs (DMARDs) without adequate response, or that treatment with DMARDs has been discontinued due to intolerance.

The aim of this study was to examine the effect of socioeconomic class and immigrant status on the outcome in RA, in a longitudinal observational study of early RA in Sweden (BARFOT). Our hypothesis was that lower socioeconomic class and immigrant status would have a negative effect on outcome.

\section{METHODS}

During the period 1992-2006, 2,800 patients were enrolled in the BARFOT (Better Anti-Rheumatic FarmacOTherapy) study, a multi-centre longitudinal observational study of patients with early RA in southern Sweden [8-10]. In this study, all patients had a disease duration of $\leq 2$ years, were $>18$ years of age and fulfilled the American College of Rheumatology RA classification criteria from 1987 [11]. The disease activity was evaluated at inclusion, at 3, 6 and 12 months, and at 2, 5 and 8 years. The number of swollen joints (28-joint count) (SJC), the number of tender joints (28-joint count) (TJC), C-reactive protein (CRP) levels, erythrocyte sedimentation rate (ESR), the Swedish version of the Stanford Health Assessment Questionnaire (HAQ) [12, 13], and visual analog scale (VAS) for pain and general health were measured on every follow-up occasion. The Disease Activity Score using 28joint count (DAS28) was calculated at inclusion and on every follow-up occasion (www.das-score.nl). The disease duration was calculated from the start of the symptoms.

EULAR (European League Against Rheumatism) response was calculated from the DAS28 scores [14]. DAS28 is a composite score consisting of the number of swollen joints (of 28), the number of tender joints (of 28), ESR, and the patient's global assessment (www.dasscore.nl). The patients were classified into three EULAR response groups: no response, moderate response or good response. To be a good responder, a patient had to show an improvement of at least 1.2 units and achieve an absolute score of $<3.2$. Non-responders had to show an improvement of $<0.6$, or $>0.6$ and $\leq 1.2$, and have a final DAS28 score of $>$ 5.1. Moderate responses fell in-between these criteria. Rheumatoid factor (RF) was analysed at inclusion in the study. Treatment with DMARDs and glucocorticoids was registered at inclusion and at each follow-up point. The cumulative number of DMARDs and biologics was assessed in the 2010 postal questionnaire. The choice of DMARD treatment in the BARFOT study was left to the discretion of the rheumatologist.

\section{Self-Completion Postal Questionnaire in 2010}

Between March and September 2010, all patients who were still alive in the BARFOT study $(n=2,102)$ received a self-completion postal questionnaire assessing smoking, pack-years, use of snuff, second-hand exposure to tobacco smoke, alcohol use, diet, pain, medication, co-morbidities, height, weight, waist circumference, physical activity and health-related quality of life (HRQL) as measured by the EuroQol [15]. Smoking data used in this article was assessed at inclusion in the study (never, previous, current smokers). Demographics such as main occupation and immigrant status (first-generation) were also recorded. The occupations were then coded according to the latest version of the socioeconomic status designation (Socioekonomisk indelning, SEI) in Sweden. In this study, SEI status was recorded as blue-collar worker, upper or lower white-collar worker, other, or self-employed. The group "other" in the SEI consisted of students, housewives and other people with no occupation. Data on smoking, stopping smoking, secondhand exposure to smoke and the use of snuff and alcohol have been published [16-21]. Data on diet, pain and physical activity will be published separately. Body mass index (BMI) data were available through the 2010 questionnaire, the patient records and the BARFOT database, and has been validated and published [22].

Two reminders were sent to the patients who had not responded to the first and second mailing of the selfcompleted questionnaire in 2010. All patients received written information about the self-completion postal questionnaire in 2010, and the ethics committee of Lund University approved the BARFOT study and the postal questionnaire in 2010.

\section{Statistics}

The Mann-Whitney test and chi-square test were used when assessing differences in disease activity variables and EULAR response between immigrants and non-immigrants. For the differences between the different socioeconomic classes, Kruskall-Wallis test was used.

Multiple logistic regression analyses were performed using as outcome EULAR good response vs no or moderate response at 5 and 8 years. The following variables were entered in the regression analyses: baseline age, baseline disease duration (months), sex, baseline HAQ, socioeconomic class (SEI class), immigrant status (yes/no), $\mathrm{RF}$, baseline smoking (never-smokers, previous smokers or current smokers), number of previous DMARDs and biologics (grouped together), and body mass index (BMI) at baseline. SEI class and the number of previous DMARDs and biologics were from the 2010 questionnaire. The variables entered in the regression models were checked for co-linearity. The reference for SEI class was upper whitecollar, for immigration status it was being an immigrant, for smokers it was never having smoked and for BMI it was 1925.9. IBM SPSS statistics 19 was used in the statistical analyses.

\section{RESULTS}

Altogether, 1,460 patients who had answered the 2010 self-completion postal questionnaire and who were $>18$ years of age and had a disease duration of $\leq 24$ months were included in this study. Patients who did not answer the 2010 questionnaire $(579$ of $2,104,27.5 \%)$ had higher mean DAS28 (5.4 for non-responders $v s 5.2$ for responders, $\mathrm{p}=$ $0.01)$, higher mean VAS health $(48 \mathrm{~mm}$ vs $45 \mathrm{~mm}, \mathrm{p}=$ $0.008)$, and higher mean number of SJCs (11 vs 10, $\mathrm{p}=0.03)$ at inclusion, were more often smokers $(30 \%$ vs $24 \%, \mathrm{p}=$ 
$0.01)$ and were less often RF-positive $(58 \%$ vs $63 \%, \mathrm{p}=$ 0.02) than the patients who had answered the 2010 questionnaire [18].

The demographics and baseline disease characteristics stratified for socioeconomic class and immigration status are shown in Table 1. Socioeconomic class data were available for 1,158 out of $1,460(79 \%)$ of the patients. A total of 511 $(44.1 \%)$ were blue-collar workers, 526 (45.4\%) were lower white-collar workers, $81(7.0 \%)$ were upper white-collar workers, 16 (1.4\%) were self-employed, and 24 (2.1\%) were in the class "other" (i.e. students, housewives and others with no occupation). Patients who were self-employed were older. Blue-collar workers and patients in the SEI class "other" had more pain, but there were no other significant differences in disease activity at baseline. A higher proportion of bluecollar workers and those who were self-employed smoked. There were no differences in disease duration $(\mathrm{p}=0.25), \mathrm{RF}$ $(\mathrm{p}=0.41)$ or anti-CCP status $(\mathrm{p}=0.65)$, stratified according to SEI class.

\section{Socioeconomic Status and Outcome}

There were no significant differences in glucocorticoid treatment and treatment with DMARDs and biologics at the different follow-up points, stratified for SEI class (data not shown). When looking at the cumulative mean number of previous DMARDs and biologics (grouped together) in the 2010 questionnaire, the figures were 1.7 for blue-collar workers, 1.9 for lower white-collar workers, 1.8 for upper white-collar workers, 1.2 for people who were self-employed and 1.3 for others (overall $\mathrm{p}=0.01$ ). There were no significant differences in the number of current DMARDs and biologics (grouped together), stratified for SEI class.

There were few significant differences in HAQ, DAS28, VAS pain and global health, SJC, TJC, ESR, and CRP for up to 8 years of follow-up, stratified for SEI class (data not shown).

There were no significant differences in EULAR response, stratified for SEI class, for up to 8 years of followup $(\mathrm{p}=0.72, \mathrm{p}=0.47, \mathrm{p}=0.68, \mathrm{p}=0.79, \mathrm{p}=0.12, \mathrm{p}=0.25$ for 3,6 and 12 months, and 2, 5 and 8 years, respectively). This did not change when looking at non-immigrants only. There were no differences in the absolute DAS28 values up to 8 years of follow-up between the different SEI classes (data not shown).

\section{Immigrant Status and Outcome}

One hundred thirty-nine of 1,430 patients $(9.7 \%)$ were immigrants and 77 of 132 of the immigrants (58\%) were from other Scandinavian countries, mainly Finland and Denmark. Data on immigration status was missing in 30 patients. Data on which country the patient had immigrated from was available for 132 patients. There were no significant differences in gender, age at inclusion or disease duration at inclusion between immigrants and nonimmigrants. More immigrants than non-immigrants were in the social class "other" (standardized residual 2.0, overall $\mathrm{p}$

Table 1. Disease Activity at Inclusion and Demographics Stratified for Socioeconomic Class and Immigration Status. Data are Mean (SD) Unless Otherwise Indicated. This Data is from the BARFOT Study at Inclusion

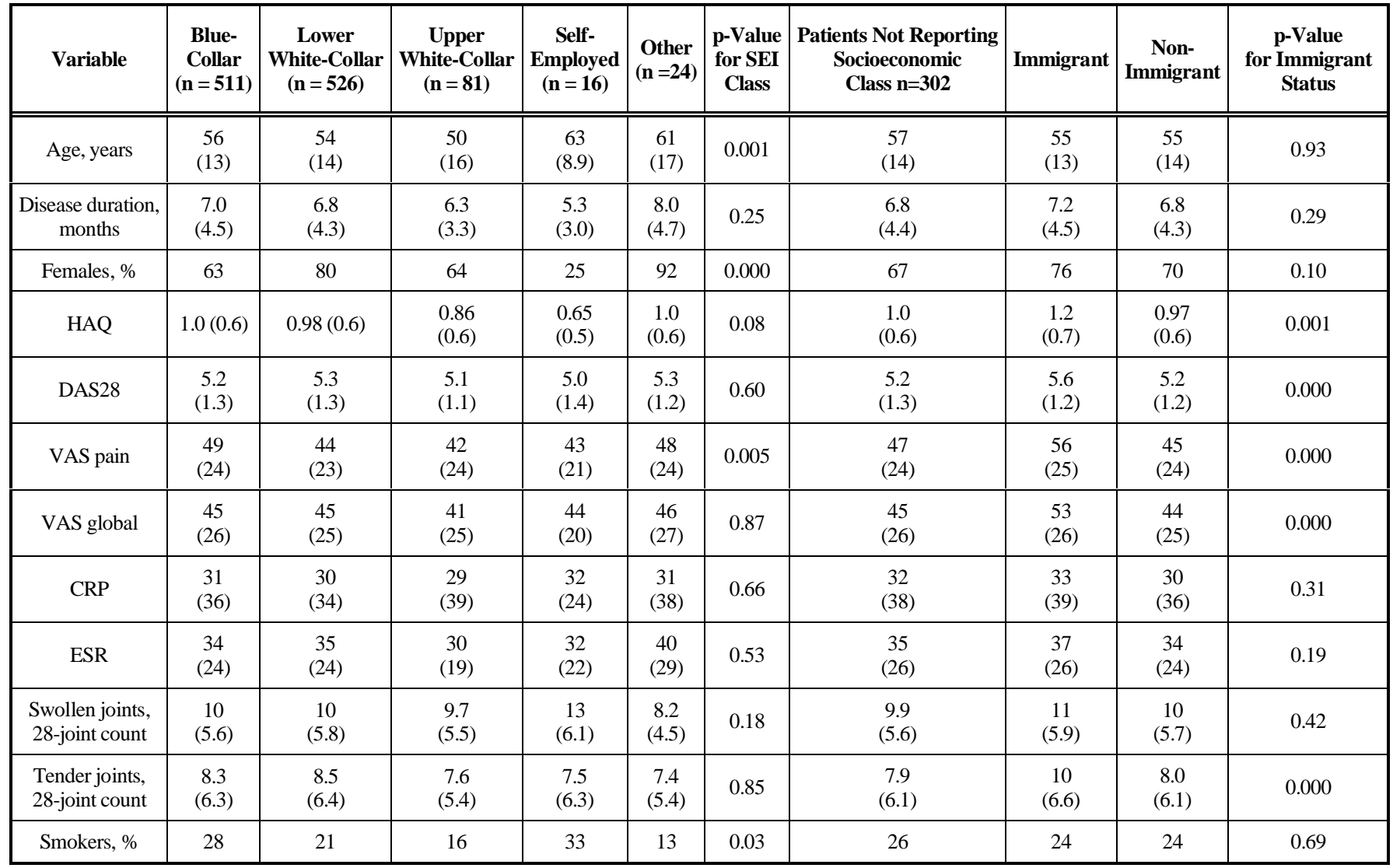


$=0.02)$. At inclusion, there were no significant differences in smoking status between immigrants and non-immigrants ( $\mathrm{p}$ $=0.69)$, RF $(\mathrm{p}=0.19)$ or anti-CCP status $(\mathrm{p}=0.20)$. AntiCCP data were available for 388 patients.

There were no significant differences in the cumulative mean number of previous or current DMARDs or cumulative number of current or previous biological treatments, as assessed by the 2010 questionnaire, between immigrants and non-immigrants (data not shown). However, immigrants more often received DMARD treatment at the first visit (87\% immigrants vs $79 \%$ non-immigrants (standardized residual -2.0$)$ and more often received combination treatment with DMARDs at the first visit $(4.3 \%$ vs $1.3 \%$, standardized residual 2.5, overall $\mathrm{p}=0.02$ ). There were no other significant differences in DMARD treatment later in monotherapy or combination therapy, in treatment with methotrexate or sulphasalazine, or in treatment with biologics. There were no significant differences in treatment with glucocorticoids at any time point between immigrants and non-immigrants (data not shown).

At inclusion, immigrants had significantly higher mean HAQ (immigrants 1.2 vs non-immigrants $0.97, \mathrm{p}=0.001$ ), higher mean DAS28 (5.6 vs 5.2, p =0.000), higher mean VAS pain ( $56 \mathrm{~mm}$ vs $45 \mathrm{~mm}, \mathrm{p}=0.000)$, higher mean VAS global health $(53 \mathrm{~mm} v s 44 \mathrm{~mm}, \mathrm{p}=0.000)$ and higher mean TJC (10 vs 8, $\mathrm{p}=0.000)$. These differences persisted at all follow-up points up to 2 years of follow-up (data not shown). At five years, immigrants had significantly higher mean HAQ (0.69 vs 0.56, p=0.04), DAS28 (3.2 vs 3.0, $\mathrm{p}=0.048$ ) and VAS pain (32 mm vs $27 \mathrm{~mm}, \mathrm{p}=0.02)$. At 8 years, immigrants had significantly higher mean HAQ (0.8 vs $0.6, \mathrm{p}$ $=0.02)$. Immigrants also scored significantly worse than non-immigrants in mean EuroQol score in the 2010 questionnaire $(0.64$ vs $0.73, \mathrm{p}=0.000)$. However, for CRP, ESR and SJC, there were no statistically significant significances at any time point, stratified for immigration status (data not shown).

There were no significant differences in EULAR response between immigrants and non-immigrants $(p=0.63$, $\mathrm{p}=0.81, \mathrm{p}=0.11, \mathrm{p}=0.47, \mathrm{p}=0.07, \mathrm{p}=0.53$ for 3,6 and 12 months, and 2, 5 and 8 years, respectively). This did not change when we only considered immigrants, stratified for SEI class. Immigrants scored higher than non-immigrants in absolute DAS28 values up to 5 years of follow-up $(\mathrm{p}=0.001$, $\mathrm{p}=0.19, \mathrm{p}=0.008, \mathrm{p}=0.003, \mathrm{p}=0.048$ for 3,6 and 12 months, and 2 and 5 years, respectively).

\section{Patients who Immigrated from Scandinavian Countries vs Other Countries}

Seventy-seven of 132 patients $(58 \%)$ had immigrated to Sweden from other Scandinavian countries and 55 of the 132 (42\%) had come from outside Scandinavia. Immigrants from non-Scandinavian countries more often received glucocorticoids at inclusion and at 3 and 6 months than immigrants from Scandinavian countries ( $42 \%$ vs $23 \%$ at inclusion, $\mathrm{p}=0.02$; $47 \%$ vs $27 \%$ at 3 months, $\mathrm{p}=0.02$; and $44 \%$ vs $25 \%$ at 6 months, $\mathrm{p}=0.03$ ) but these differences did not persist in the follow-up. There were no significant differences in DMARD treatment or treatment with biologics at the follow-up points, but in the 2010 questionnaire immigrants from non-
Scandinavian countries had received more DMARDs and biologics cumulatively $(2.0 \mathrm{vs} 1.8, \mathrm{p}=0.04)$. There were no significant differences in RF ( $\mathrm{p}=0.87)$, smoking status $(\mathrm{p}=$ $0.31)$, SEI class $(\mathrm{p}=0.07)$ or anti-CCP status $(\mathrm{p}=0.93)$ between immigrants from Scandinavia and immigrants from non-Scandinavian countries.

There were several statistically significant differences in disease activity between immigrants from non-Scandinavian countries and immigrants from other countries in Scandinavia. At inclusion, the immigrants from non-Scandinavian countries had significantly more pain $(62 \mathrm{~mm}$ vs $53 \mathrm{~mm}, \mathrm{p}=0.03)$ and higher VAS global (61 vs 48, $\mathrm{p}=0.003)$, but lower CRP (27vs $37, \mathrm{p}=0.03)$ and lower $\operatorname{ESR}(30 v s \mathrm{41}, \mathrm{p}=0.009)$. At 3 months, the immigrants from non-Scandinavian countries had higher HAQ (0.9 vs 0.6, p = 0.04), higher DAS28 (4.5 vs 4.0, p = 0.02), higher pain (44 vs 31, p=0.01), worse VAS global (42 vs 30, $\mathrm{p}$ $=0.046)$, higher SJC (7 vs 5, p = 0.04) and higher TJC (8vs 5, p $=0.005)$. However, these significant differences did not persist in the follow-up, and there were few significant differences up to 8 years of follow-up (data not shown). EQ-5D values in the 2010 questionnaire did not differ significantly between immigrants from Scandinavia and immigrants from nonScandinavian countries $(\mathrm{p}=0.25)$.

The patients who had emigrated from non-Scandinavian countries did not have significantly different EULAR responses from patients who had emigrated from other Scandinavian countries, with the exception of EULAR response at 5 years, where $24 \%$ on non-Scandinavians had no EULAR response, as compared to $8 \%$ of Scandinavians (overall $\mathrm{p}=0.01$ ).

\section{Multiple Logistic Regression Analyses}

We performed multiple logistic regression analyses to study which factors were independently associated with EULAR outcome. The outcome was EULAR good response versus no or moderate response at 5 and 8 years (Table 2).

Immigrant status was not independently associated with good EULAR outcome. SEI class "other", longer disease duration, female sex, current smoking at inclusion, and more cumulative treatment with DMARDs and biologics were independently associated with poorer EULAR response at 5 years of follow-up. At 8 years, higher age at inclusion, more cumulative treatment with DMARDs and biologics, female sex, RF positivity and current smoking at inclusion were associated with poorer EULAR response, and higher HAQ at inclusion with better response.

\section{Attrition}

EULAR response data were available for 1,381 patients at 3 months, and 1,285,1,342,1,319,1,295 and 795 patients at 6 and 12 months and 2, 5 and 8 years, respectively. There were no significant differences in the number of patients with complete EULAR data, stratified for SEI class $(\mathrm{p}=0.69)$ or immigrant status $(\mathrm{p}=0.08)$.

\section{DISCUSSION}

To our knowledge, this is the first study to investigate the effect of immigrant status on treatment and outcome in RA in Sweden. The study showed that first-generation 
Table 2. Multiple Logistic Regression Analysis with EULAR Good Response vs No or Moderate Response at 5 and 8 Years of Follow-Up. The Reference for SEI Class was Upper White-Collar, for Immigration Status it was Being an Immigrant, for Smokers it was Never Having Smoked and for BMI it was 19-25.9

\begin{tabular}{|c|c|c|c|c|c|c|}
\hline Variable & \multicolumn{3}{|c|}{5 Years of Follow-Up } & \multicolumn{3}{|c|}{8 Years of Follow-Up } \\
\hline Disease duration, months & 0.95 & $0.92-0.99$ & 0.02 & 1.0 & $0.95-1.06$ & 0.88 \\
\hline Male sex & 2.23 & $1.50-3.32$ & 0.000 & 2.85 & $1.74-4.68$ & 0.000 \\
\hline Lower white-collar & 0.91 & $0.47-1.77$ & 0.77 & 0.98 & $0.42-2.29$ & 0.97 \\
\hline Self-employed & 0.76 & $0.18-3.27$ & 0.71 & 0.78 & $1.13-4.73$ & 0.78 \\
\hline Other & 0.15 & $0.04-0.67$ & 0.01 & 0.52 & $0.11-2.49$ & 0.41 \\
\hline Non-immigrant & 0.84 & $0.43-1.65$ & 0.62 & 0.78 & $0.32-1.86$ & 0.57 \\
\hline Cumulative number of previous DMARDs and biologics & 0.64 & $0.56-0.74$ & 0.000 & 0.63 & $0.54-0.74$ & 0.000 \\
\hline HAQ & 1.26 & $0.95-1.69$ & 0.11 & 2.01 & $1.38-2.93$ & 0.000 \\
\hline BMI $<19$ & 0.94 & $0.30-2.92$ & 0.91 & 0.83 & $0.22-3.10$ & 0.78 \\
\hline BMI 26-30 & 0.98 & $0.67-1.44$ & 0.91 & 0.70 & $0.44-1.13$ & 0.14 \\
\hline $\mathrm{BMI}>30$ & 0.76 & $0.47-1.24$ & 0.27 & 0.59 & $0.33-1.05$ & 0.07 \\
\hline
\end{tabular}

immigrants in Sweden, as compared to non-immigrants, had worse scores in VAS pain, TJC, HAQ, and VAS global, and higher DAS28 at baseline, and these findings persisted for up to 2 years of follow-up - and for HAQ even up to 8 years of follow-up. Immigrants also scored worse than nonimmigrants in HRQL, as measured by the EuroQol in the 2010 questionnaire. However, immigrant status did not have any effect on EULAR outcome or objective measures of inflammation such as SJC, ESR and CRP. Immigrants from outside Scandinavia had higher disease activity initially than immigrants from other Scandinavian countries, but these differences disappeared after the first 3 months of follow-up. There were small differences in anti-rheumatic treatment, in particular that immigrants from non-Scandinavian countries received more glucocorticoids during the first 6 months than immigrants from Scandinavia, and these patients had also received a higher number of DMARDs and biologics cumulatively over time. Thus, immigrants did not have a poorer EULAR response.

We suspect that the predominant symptom in immigrants could be pain. Immigrants scored consistently higher than non-immigrants in VAS pain and TJC up to 2 years, and this may have affected also the DAS28, HAQ and VAS global, and it may even have affected the EuroQol. One explanation for these results could also be cultural differences in pain perception or expression, or stress due to immigration or comorbidities. Two studies from the US report a negative effect of minority status on outcome in RA $[6,7]$. The study by Barton et al. from the US reported higher DAS28 and poorer function in non-White groups and also in immigrants and non-English language groups in a university clinic but could show no such differences in a public county hospital. The authors speculate on the reasons for this, for example communication barriers and inaccurate understanding, genetic and biologic differences, patient behaviour and delivery system structure [7]. These findings on immigrants scoring worse in pain, ADL function and HRQL may help clinicians to focus on these factors.

The other main finding of this study was that socioeconomic class did not have any impact on treatment or outcome in RA in Sweden, in contrast to earlier reports from Canada, the Netherlands and the UK on the negative effect of lower socioeconomic class on outcome in RA [1-3]. Thus, our hypothesis of lower socioeconomic status correlating with poorer outcome in RA did not hold true. In Sweden, access to healthcare is universal, and prescription drugs are subsidised by the state. This means, for example, that biological treatment for RA in Sweden has been and is now (year 2013) entirely free of charge for the patients. Swedish society differs from both the UK and the USA in particular, in that social differences are not so marked and that access to healthcare is not dependent on social class, employment status or whether or not one has private insurance. We defined socioeconomic class by main occupation. We realise that the work profile in several occupations has changed over time, which often means physically less demanding work nowadays. However, we could see differences in lifestyle factors such as smoking between the different socioeconomic classes.

The SEI class "other" emerged as an independent factor associated with poorer EULAR response at 5 years of follow-up, but otherwise we could not see that SEI class was 
independently associated with EULAR outcome in the regression analyses. The SEI group "other" was small and consisted of students, housewives and other people with no occupation, and this finding needs to be confirmed in a larger material. Immigrant status was not independently associated with EULAR outcome at 5 or 8 years of followup. Female sex, longer disease duration, current smoking at inclusion and a higher number of previous DMARDs and biologics were associated with poor response at five years. At 8 years of follow-up, longer disease duration, female sex, RF positivity, current smoking at inclusion and higher number of cumulative treatment were also associated with poorer prognosis. We think that the association between more treatment and poorer prognosis could be due to confounding by indication, as more treatment probably is a marker for a more aggressive disease.

The main strength of the study was the large, welldocumented RA cohort with a comprehensive follow-up and a long follow-up of 8 years. We could not detect differences in attrition between the different socioeconomic classes or stratified for immigrant status. We classified patients socioeconomically according to their main occupation in life, and we may therefore have misclassified some patients. We did not record race or ethnicity in this study. Also, we do not have data on co-morbidities, which could affect pain, EuroQol, HAQ and global health. We knew the anti-CCP status of only a minority of patients. The cumulative number of treatment with DMARDs and biologics was assessed in the 2010 postal questionnaire, and can thus be susceptible to problems with recall.

In conclusion, to our knowledge this is the first study to investigate the effect of immigrant status on anti-rheumatic treatment and treatment response in RA in Sweden. This is also the first study to look at the effects of socioeconomic class on outcome in RA in Sweden. Immigrants scored worse in VAS pain, TJC, HAQ, VAS global, and DAS28 than non-immigrants, and these differences persisted for up to 5 years of follow-up - and for HAQ, up to 8 years. Immigrants also scored worse in HRQL as measured by the EuroQol cross-sectionally in 2010 , as compared to nonimmigrants. However, immigrant status was not associated with poorer EULAR outcome or with higher SJC, ESR or CRP. We suspect that the predominant symptom in immigrants could be pain. Socioeconomic class did not have any effect on treatment or outcome in RA in this large longitudinal observational cohort of Swedish patients. There were small differences in anti-rheumatic treatment, stratified for immigration and socioeconomic class. The relatively egalitarian society in Sweden and good access to rheumatological care and treatment with DMARDs and biologics may have contributed to these results.

\section{CONFLICT OF INTEREST}

Dr. M. K. Söderlin has received speaking fees for educational events from Abbott $(<\$ 3,000)$ and MSD (< $\$ 2,000$ ), and speaking fees and consultation fees from Pfizer $(<\$ 10,000)$. Dr. Stefan Bergman has received consultation fees from Pfizer $(<\$ 5,000)$.

\section{ACKNOWLEDGEMENTS}

We thank Jan-Åke Nilsson for invaluable help with the statistical analyses. We also thank Henrik Karlsson for the SEI classification. We are grateful for the help provided by all our colleagues and staff at the hospitals contributing to the BARFOT study, for their cooperation and provision of data.

\section{REFERENCES}

[1] Harrison MJ, Tricker KJ, Davies L, et al. The relationship between social deprivation, disease outcome measures, and response to treatment in patients with stable, long-standing rheumatoid arthritis. J Rheum 2005; 32(12): 2330-6.

[2] Marra CA, Lynd LD, Esdaile JM, et al. The impact of low family income on self-reported health outcomes in patients with rheumatoid arthritis within a publicly funded health-care environment. Rheumatol 2004; 43(11): 1390-7.

[3] Jacobi CE, Mol GD, Boshuizen HC, et al. Impact of socioeconomic status on the course of rheumatoid arthritis and on related use of health care services. Arthritis Rheum 2003; 49(4): 567-73.

[4] Socioeconomic deprivation and rheumatoid disease: what lessons for the health service? ERAS Study Group. Early Rheumatoid Arthritis Study. Ann Rheum Dis 2000; 59(10): 794-9.

[5] Li X, Sundquist J, Sundquist K. Socioeconomic and occupational risk factors for rheumatoid arthritis: a nationwide study based on hospitalizations in Sweden. J Rheum 2008; 35(6): 986-91.

[6] Bruce B, Fries JF, Murtagh KN. Health status disparities in ethnic minority patients with rheumatoid arthritis: a cross-sectional study. J Rheum 2007; 34(7): 1475-9.

[7] Barton JL, Trupin L, Schillinger D, et al. Racial and ethnic disparities in disease activity and function among persons with rheumatoid arthritis from university-affiliated clinics. Arthritis Care Res 2011; 63(9): 1238-46.

[8] Forslind K, Ahlmen M, Eberhardt K, et al. Prediction of radiological outcome in early rheumatoid arthritis in clinical practice: role of antibodies to citrullinated peptides (anti-CCP). Ann Rheum Dis 2004; 63(9): 1090-5.

[9] Forslind K, Hafstrom I, Ahlmen M, et al. Sex: a major predictor of remission in early rheumatoid arthritis? Ann Rheum Dis 2007; 66(1): 46-52.

[10] Svensson B, Ahlmen M, Forslind K. Treatment of early RA in clinical practice: a comparative study of two different DMARD/corticosteroid options. Clin Exp Rheum 2003; 21(3): 327-32.

[11] Arnett FC, Edworthy SM, Bloch DA, et al. The American Rheumatism Association 1987 revised criteria for the classification of rheumatoid arthritis. Arthritis Rheum 1988, 31(3): 315-24.

[12] Ekdahl C, Eberhardt K, Andersson SI, Svensson B. Assessing disability in patients with rheumatoid arthritis. Use of a Swedish version of the Stanford Health Assessment Questionnaire. Scand J Rheumatol 1988; 17(4): 263-71.

[13] Fries JF, Spitz P, Kraines RG, et al. Measurement of patient outcome in arthritis. Arthritis Rheum 1980; 23(2): 137-45.

[14] van Gestel AM, Haagsma CJ, van Riel PL. Validation of rheumatoid arthritis improvement criteria that include simplified joint counts. Arthritis Rheum 1998; 41(10): 1845-50.

[15] EuroQol--a new facility for the measurement of health-related quality of life. The EuroQol Group. Health Policy 1990, 16(3):199208.

[16] Soderlin MK, Bergman S. Absent "Window of Opportunity" in smokers with short disease duration. Data from BARFOT, a multicenter study of early rheumatoid arthritis. J Rheum 2011; 38(10): 2160-8.

[17] Soderlin MK, Petersson IF, Bergman S, et al. Smoking at onset of rheumatoid arthritis (RA) and its effect on disease activity and functional status: experiences from BARFOT, a long-term observational study on early RA. Scand J Rheumatol 2011; 40(4): 249-55.

[18] Andersson ML, Bergman S, Soderlin MK. The effect of snuff (smokeless tobacco) on disease activity and function in rheumatoid arthritis: experiences from the Better Anti-Rheumatic 
FarmacOTherapy, a longitudinal multicenter study on early rheumatoid arthritis. J Clin Rheumatol 2013; 19(1): 14-8.

[19] Soderlin MK, Andersson M, Bergman S. Second-hand exposure to tobacco smoke and its effect on disease activity in Swedish rheumatoid arthritis patients. Data from BARFOT, a multicenter study of RA. Clin Exp Rheum 2013; 31(1): 122-4.

[20] Bergman S, Symeonidou S, Andersson MLE, et al. Moderate and heavy alcohol consumption is associated with less inflammation and better health-related quality of life in female rheumatoid arthritis patients in Sweden. Data from BARFOT, a multicenter study on early RA. BMC Musculoskeletal Disord 2013; 24(14): 218.

[21] Andersson ML, Bergman S, Soderlin MK. The Effect of Stopping Smoking on Disease Activity in Rheumatoid Arthritis (RA). Data from BARFOT, a Multicenter Study of Early RA. Open Rheumatol J 2012; 6: 303-9.

[22] Ajeganova S, Andersson ML, Hafstrom I. Obesity is associated with worse disease severity in rheumatoid arthritis as well as with co-morbidities - a long-term follow-up from disease onset. Arthritis Care Res 2013; 65 (1): 78-87.

(C) Andersson et al.; Licensee Bentham Open.

This is an open access article licensed under the terms of the Creative Commons Attribution Non-Commercial License (http://creativecommons.org/licenses/by-nc/ 3.0/) which permits unrestricted, non-commercial use, distribution and reproduction in any medium, provided the work is properly cited. 